\title{
Influence of patient age on refraction and corneal haze after photorefractive keratectomy
}

\author{
L Hefetz, Y Domnitz, D Haviv, D Krakowsky, Y Kibarsky, S Abrahami, P Nemet
}

\begin{abstract}
Aims/background-Since wound healing processes are known to be more rapid in those who are young, it was decided to examine the effect of patient age on refractive outcome of photorefractive keratectomy (PRK).

Methods-The records of 599 eyes that had undergone PRK were studied retrospectively. The eyes were categorised by baseline myopia and patient age. Spherical equivalent and corneal haze were compared in the baseline refraction and age groups at 3, 6, and 12 months after PRK.

Results-There were no differences in postoperative refraction and corneal haze in the different age groups.

Conclusion-Patient age had no statistically significant effect on refraction and corneal haze 1 year after PRK.

(Br F Ophthalmol 1997;81:637-638)
\end{abstract}

Photorefractive keratectomy (PRK) appears to offer an attractive alternative to spectacles for all age groups. During the past 5 years PRK results have been encouraging, especially in low and moderate myopes, ${ }^{1-3}$ although the reasons remain unclear. We undertook to study whether patient age might be one of the factors affecting the outcome of PRK.

Table 1 Number of eyes in each group

\begin{tabular}{clll}
\hline \multirow{4}{*}{} & \multicolumn{3}{l}{ Baseline refraction (D) } \\
\cline { 2 - 4 } & -2.00 to -6.00 & -6.25 to -10.00 & $\geqslant-10.25$ \\
\hline Age (years): & & & \\
$<30$ & 149 & 82 & 21 \\
$30-39$ & 102 & 79 & 19 \\
$\geqslant 40$ & 66 & 58 & 23 \\
Total & 317 & 219 & 63 \\
\hline
\end{tabular}

Ophthalmology, Assaf

Harofeh Medical

Centre, and Sackler

Faculty of Medicine,

Tel Aviv University,

Israel

L Hefetz

Y Domnitz

D Haviv

D Krakowsky

Y Kibarsky

S Abrahami

P Nemet

Correspondence to:

L Hefetz, MD, Department

of Ophthalmology, Assaf

Harofeh Medical Centre,

Zerifin 70300, Israel.

Accepted for publication 26 March 1997

${ }^{\star} \mathrm{p}$ value comparing these dioptres was 0.01 according to analysis of variance. $t \mathrm{p}$ value comparing these dioptres was 0.01 according to analysis of variance. $\neq \mathrm{p}$ value comparing these dioptres was 0.02 according to analysis of variance.

\section{Material and methods}

We studied retrospectively the medical records of 599 eyes that had undergone PRK (Aesculap-Meditec, Mel 60, Germany) in our outpatient clinic and had a complete postoperative follow up of 1 year. The eyes were classified into three groups according to preoperative baseline refraction (spherical equivalent): group 1 , low myopes $(-2.00 \mathrm{D}$ to $-6.00 \mathrm{D})$; group 2 , moderate myopes $(-6.25 \mathrm{D}$ to -10.00 D); group 3, high myopes (-10.25 D and greater). Each refraction group was divided into three age groups: under 30 years (age group 1); 30 to 39 years (age group 2); and 40 years and older (age group 3). Spherical equivalent and anterior corneal haze were measured 3, 6, and 12 months after PRK. Corneal haze was graded on a scale of 0 to +4 (clear cornea to dense scar). The results were analysed by analysis of variance.

\section{Results}

Table 1 shows the number of eyes studied in each group. Table 2 summarises the spherical equivalent in each group at 3, 6, and 12 months after PRK. After 12 months, residual myopia in the low myopic group was -0.24 (SD 0.68) D, $-0.46(0.92) \mathrm{D}$, and -0.28 (0.81) D in the three age groups, respectively $(\mathrm{p}=0.08)$; in the moderate myopic group it was -0.70 (0.81) D, -0.87 (0.90) D, and -0.81 (0.97) $\mathrm{D}$ in the three age groups $(\mathrm{p}=0.55)$; and in the high myopic group it was $-1.51(1.4) \mathrm{D}$, $-1.63(1.9) \mathrm{D}$, and -1.54 (1.32) $\mathrm{D}$ in the three age groups, respectively $(\mathrm{p}=0.68)$.

Table 3 summarises the degree of anterior corneal haze in each baseline refraction and age group at 3, 6, and 12 months after PRK. Haze degree, after 1 year in the low myopic group was $0.26(0.48), 0.29(0.49)$, and 0.34 (0.54) in age groups 1,2 , and 3 , respectively $(\mathrm{p}=0.48)$; in the moderate myopic group it was

Table 2 Spherical equivalent after photorefractive keratectomy (PRK) in each age and baseline refraction group

\begin{tabular}{|c|c|c|c|c|c|}
\hline \multirow[b]{3}{*}{ Baseline refraction group } & \multirow[b]{3}{*}{ Time after PRK (months) } & \multicolumn{3}{|c|}{ Spherical equivalent } & \multirow[b]{3}{*}{$p$ Value } \\
\hline & & \multicolumn{3}{|c|}{ Age (years) } & \\
\hline & & $<30$ & $30-39$ & $\geqslant 40$ & \\
\hline \multirow[t]{3}{*}{1 Low myopes } & 3 & -0.03 & -0.15 & +0.12 & 0.08 \\
\hline & 6 & -0.07 & -0.20 & +0.01 & 0.24 \\
\hline & 12 & $-0.24^{\star}$ & $-0.46 \dagger$ & $-0.28 \ddagger$ & 0.08 \\
\hline \multirow[t]{3}{*}{2 Moderate myopes } & 3 & -0.22 & -0.51 & -0.12 & 0.36 \\
\hline & 6 & -0.33 & -0.62 & -0.40 & 0.42 \\
\hline & 12 & $-0.70^{\star}$ & $-0.87 \dagger$ & $-0.81 \ddagger$ & 0.55 \\
\hline \multirow[t]{3}{*}{3 High myopes } & 3 & -0.48 & -0.10 & -0.91 & 0.39 \\
\hline & 6 & -0.64 & -1.44 & -1.39 & 0.27 \\
\hline & 12 & $-1.51^{\star}$ & $-1.63 \dagger$ & $-1.54 \ddagger$ & 0.68 \\
\hline
\end{tabular}


Table 3 Anterior corneal haze after photorefractive keratectomy (PRK) in each age and baseline refraction group

\begin{tabular}{|c|c|c|c|c|c|}
\hline \multirow[b]{3}{*}{ Baseline refraction group } & \multirow{3}{*}{$\begin{array}{l}\text { Time after PRK } \\
\text { (months) }\end{array}$} & \multicolumn{3}{|c|}{ Anterior corneal haze } & \multirow[b]{3}{*}{$p$ Value } \\
\hline & & \multicolumn{3}{|c|}{ Age (years) } & \\
\hline & & $<30$ & $30-39$ & $\geqslant 40$ & \\
\hline \multirow[t]{3}{*}{1 Low myopes } & 3 & 0.78 & 0.78 & 0.86 & 0.70 \\
\hline & 6 & 0.56 & 0.52 & 0.48 & 0.59 \\
\hline & 12 & $0.26^{\star}$ & $0.29 \dagger$ & $0.34 \ddagger$ & 0.49 \\
\hline \multirow[t]{3}{*}{2 Moderate myopes } & 3 & 1.15 & 0.89 & 0.94 & 0.45 \\
\hline & 6 & 0.85 & 0.86 & 0.78 & 0.75 \\
\hline & 12 & $0.53^{\star}$ & $0.57 \dagger$ & $0.54 \ddagger$ & 0.92 \\
\hline \multirow[t]{3}{*}{3 High myopes } & 3 & 1.52 & 1.26 & 1.26 & 0.38 \\
\hline & 6 & 1.19 & 1.05 & 1.10 & 0.85 \\
\hline & 12 & $0.66^{\star}$ & $0.78 \dagger$ & $1.15 \ddagger$ & 0.07 \\
\hline
\end{tabular}

${ }^{\star} \mathrm{p}$ value comparing these haze degrees was 0.0001 according to analysis of variance. tp value comparing these haze degrees was 0.0009 according to analysis of variance.

$\ddagger \mathrm{p}$ value comparing these haze degrees was 0.00001 according to analysis of variance.

0.53 (0.59), 0.57 (0.65), and 0.54 (0.59) in the three age groups $(\mathrm{p}=0.92)$; and in the high myopic group it was $0.66(0.65), 0.78(0.70)$, and $1.15(0.72)$ in the three age groups $(\mathrm{p}=0.07)$.

\section{Discussion}

Since both myopic regression and corneal haze are known to depend on wound healing processes which occur more rapidly in younger individuals, ${ }^{4}$ it was expected that this group would exhibit greater myopic regression and corneal haze following PRK than the adult group. However, our retrospective findings demonstrated that neither the amount of residual myopia nor the degree of anterior corneal haze were affected by patient age at 3, 6, and 12 months after PRK in the three different baseline myopic groups.
The literature varies on this subject. Ditzen et $a l^{5}$ reported that patients $30-50$ years of age had a higher degree of regression than younger or older patients, while age did not affect the degree of haze. These results could not be compared with ours since the investigators did not classify their subjects by baseline myopia. Dutt et $a l^{1}$ found a significantly greater effect of PRK with larger residual hyperopia in patients over 40 years of age. The reason for the discrepancy between their results and ours is not clear.

The amount of residual myopia and degree of corneal haze were highest in the high myopic group in all age groups in our study 12 months following PRK. These results were statistically significant (Tables 2 and 3) and are in agreement with others. ${ }^{23}$

Further research with a larger sample and additional variables is required to confirm these findings, and to determine the exact mechanisms responsible for the early and late outcomes of PRK.

1 Dutt S, Seinert RF, Raizman MB, Puliafito CA. One-year results of excimer laser photorefractive keratectomy for low

2 Epstein D, Fagerholm P, Hamberg-Nystrom H, Tengroth B. Twenty-four-month follow-up of excimer laser photorefractive keratectomy for myopia. Refractive and visual acuity results. Ophthalmology 1994;101:1558-63.

3 Brancato R, Tavola A, Carones F, Scialdone A, Gallus G, Garancini P. Excimer laser photorefractive keratectomy for myopia; results of 1165 eyes. Italian Study Group. Refract Corneal Surg 1993;9:95-104.

4 Tasman W, Jaeger E, eds. Duane's clinical ophthalmology. Vol 6. Philadelphia: J B Lippincott, 1993:18.

5 Ditzen K, Anschutz T, Schroder E. Photorefractive keratectomy to treat low, medium and high myopia: a multicenter study. F Cataract Refract Surg 1994;20(suppl):234-8. 